\title{
Some Observations on the Translation of Legal Terminology in the Albanian Language
}

\section{Svjetllana Titini}

\author{
Universiteti i Durrësit “Aleksandër Moisiu” (Albania); svjetllana.titini@yahoo.com
}

\author{
Doi:10.5901/ajis.2015.v4n3p109
}

\section{Abstract}

The translation of legal terminology faces various difficulties that affect the comprehensibility of legal text in the receptive language. This article will analyse the major problems related to the translation of legal terminology, by pointing out various errors that occur during this process through examples in Albanian. The problems associated with the translation of legal terminology will be analysed in two separate groups. First the problems that arise from the specificity of International Law will be analysed. The existence of different legal orders constitutes one of the main causes from where the controversy of concepts and legal institutions or their absence in different orders originates. Each state has its own legal system, therefore its own legal terminology, means that many referents (concepts and institutes) do not have equivalent terms in the language they enter. From this fact translation errors arise related to wrong neologisms or the use of terms that express only partially the meaning of the terms of the language from which they were borrowed. Secondly, the problems arising from language will be analysed, referring to the relationship between the word and concept that varies in different languages. In this case, examples will highlight the positive or negative connotations of some terms, which have affected the choices of terminology in various occasions and in different periods of time. We will also emphasize through examples the ways in which the entirety of meanings of legal terms affects not only the ability to translate them but also the linguistic transparency. At the end we will present findings concerning the problems arising during the process of translation as well as the possible solutions to avoid errors encountered in the translation of legal terminology.

Keywords: legal terminology, legal terminology translation, translation errors, the comprehensibility of legal text, receptive language

\section{Introduzione}

Il crescente interesse per la terminologia giuridica è strettamente collegato alla posizione che occupa il diritto nella vita di un paese e dei suoi cittadini. La società è organizzata sulla base del diritto e agisce secondo le sue regole, per questa ragione la terminologia del diritto è diventata parte importante della comunicazione sia nei contatti diplomatici e relazioni internazionali, sia nei rapporti dei cittadini con l'amministrazione pubblica e le istituzioni giudiziarie ma anche ogni volta che si presenta la necessità di consultare un avvocato o di rivolgersi ad un notaio nell'attività di tutti i giorni. Nonostante l'estensione dell'uso della terminologia giuridica essa comporta delle difficoltà di comprensione all'interno della medesima lingua e ancora di più nel corso di traduzione da una lingua ad un'altra.

Negli ultimi decenni i problemi della traduzione della terminologia giuridica hanno attirato in modo specifico l'attenzione dei linguisti e dei traduttori/interpreti che ne hanno tratto oggetto per una molteplicità di lavori scientifici.

La specificità della traduzione giuridica si rivela in due aspetti fondamentali.

II primo è attribuibile al legame strettissimo tra diritto e lingua che si esprime nelle parole di Cortelazzo quando afferma che il diritto non si serve della lingua ma è fatto della lingua (1997:36). Ciò significa che il diritto deve la sua esistenza alla lingua attraverso la quale si concretizza. La lingua è fondamentale nel definire i concetti e gli istituti del diritto in quanto li rende distinguibili gli uni dagli altri e contemporaneamente accessibili alla comunicazione fornendo loro la forma di espressione.

Uno strettissimo legame si manifesta altresì tra l'ordinamento giuridico inteso come il diritto proprio di uno Stato e la lingua nazionale. In questo senso ogni Stato ha un suo ordinamento giuridico e quindi una propria terminologia giuridica.

Nella letteratura scientifica fino ad oggi i problemi principali legati alla traduzione della terminologia giuridica si raggruppano in: problemi che derivano dal diritto e problemi che derivano dalla lingua. Pertanto, i problemi sorgono per cause legate da un lato al carattere nazionale dell'ordinamento giuridico di ogni Stato e dall'altro alla materializzazione dei concetti giuridici in diversi sistemi linguistici.

In merito a questo duplice ordine di problemi posti dal raffronto dei termini e testi giuridici nel presente lavoro vorrei soffermarmi sulle difficoltà che si prospettano nella traduzione della terminologia giuridica da e verso la lingua albanese. 
II motivo di questa scelta è dovuto al livello della traduzione giuridica nella lingua albanese nell'ultimo ventennio documentato nei vari e numerosi testi universitari e documenti di natura giuridica e le sue conseguenze in relazione all'enorme quantità di documentazione da tradurre che coincide con l'aprirsi del paese al mondo occidentale e con il processo di armonizzazione della legislazione nazionale con quella dell'Unione Europea.

Inoltre in tema di difficoltà di traduzione in ambito giuridico questo lavoro cerca di mettere in evidenza le lacune nella pubblicazione di studi in materia e la carenza di supporti quali ad esempio dizionari giuridici aggiornati in seguito ai cambiamenti del diritto stesso.

Durante l'analisi verranno utilizzati esempi raccolti da diversi testi e documenti giuridici tradotti, ma anche esempi tratti dalla pratica della comunicazione orale. Attraverso gli esempi selezionati verranno evidenziati gli errori che si incontrano nel trovare quale termine nella lingua di arrivo mettere al posto del termine che deve essere tradotto e i fattori che incidono sulla densità della loro manifestazione.

\section{Problemi di Traduzione Giuridica Derivanti dalle Relazoni Semantiche all'interno della Lingua di Partenza o di Arrivo}

Riguardo ai problemi di traduzione nascenti dalla lingua, Sacco afferma che le vere difficoltà derivano dal fatto che il rapporto tra parola e concetto non è uguale in tutte le lingue giuridiche(Sacco, 1992).

In più, Sacco osserva che la lingua giuridica al pari delle altre lingue scientifiche, dovrebbe essere basata sulla corrispondenza tra una determinata parola e una categoria, definita in relazione all'insieme delle sue caratteristiche costruttive.

Difatti alcuni termini giuridici assumono connotazioni positive o negative che hanno inciso anche sulle scelte terminologiche in diversi casi e in diversi periodi. II termine divorzio, nel periodo che va dalla Seconda Guerra Mondiale fino agli anni '70, è stato tradotto in albanese shkurorëzim, termine che per effetto della connottazione religiosa è stato sostituito, dopo la proclamazione dell'ateismo di Stato, con il termine zgjidhje e martesës. Quest'ultimo è il termine ufficiale che viene usato tuttora nella terminologia giuridica albanese. II termine reclusione è stato tradotto in albanese heqje lirie fino all' entrata in vigore del nuovo codice penale (1995), dopodiché al suo posto viene usato dënim me burgim, un termine chiaro e univoco.

E necessario precisare che i problemi della traduzione giuridica nascenti dalla lingua derivano anche indirettamente dalle caratteristiche proprie della lingua giuridica stessa. (Longinotti, 2009)

Come spesso rilevato il linguaggio giuridico si fonda dall' incrocio del linguaggio ordinario con elementi di carattere tecnico presenti nelle leggi che regolamentano vari settori e infine con elementi giuridici veri e propri. (Rega, 2000:449)

Le principali questioni semantiche che riguardano l'omonimia, la sinonimia, e la polisemia generano dei problemi di ambiguità all'interno del diritto e nella sua traduzione. Prima di trovare il termine equivalente appropriato è necessario effettuare un' accurata analisi semantica del termine.

II termine mandato in italiano ha significati diversi. Questo termine indica nel diritto privato, il contratto con cui il mandatario si impegna a compiere uno o più atti giuridici nell'interesse del mandante dal quale ha ricevuto l'incarico, in procedura penale indica invece il provvedimento della magistratura a carico di un imputato (mandato di cattura, mandato di arresto). A ciascuno di questi significati in albanese corrisponde: porosi e urdhër arresti. II termine mandato viene usato lo stesso in albanese ma per indicare: a) nel diritto internazionale l'istituto previsto dal patto della Società delle Nazioni, allo scopo di sottoporre, dopo la Prima guerra mondiale, a una forma di governo internazionalmente regolata sia i territori che erano stati distaccatti dall'impero ottomano, sia quelli appartenuti all'impero coloniale tedesco (mandat mandato internazionale); b) nel diritto costituzionale quello conferito dagli elettori ai loro rappresentanti in Parlamento (mandato parlamentare); il principio secondo il quale l'eletto che riceve un mandato generale dagli elettori non ha alcun impegno giuridicamente vincolante nei loro confronti (mandat joimperativ - divieto di mandato imperativo). Questa distinzione nella traduzione, specialmente quella orale, molte volte non viene rispettata usando il termine mandato anche al posto di porosi e urdhër arresti.

Al termine tribunale ordinario in albanese corrispondono tre denominazioni: gjykatë e faktit, gjykatë e shkallës së parë, e gjykatë e rrethit gjyqësor. Tra questi termini il sinonimo gjykatë e rrethit gjyqësor è la denominazione ufficiale.

\section{Problemi di Traduzione Giuridica Derivanti dal Diritto: l'equivalenza Concettuale nella Traduzione Giuridica}

La traduzione giuridica risulta un lavoro delicato e complesso nel garantire il suo intento primario: quello della massima 
chiarezza nella comunicazione.

Nella pratica l'interprete deve trovare il termine adatto in breve tempo e produrre testi che forniscano ai destinatari un contenuto giuridico inalterato.

Nel processo di traduzione l'equivalenza al livello terminologico costituisce il punto di partenza nel costruire testi equivalenti in quanto la terminologia è un elemento chiave per l'organizzazione dell'informazione nei testi.

Arntz, Picht e Mayer intendono l'equivalenza come identità concettuale. Nell'ambito del diritto essi sottolineano che detto concetto, considerando le differenze insite tra gli ordinamenti, debba essere relativizzato (2004:171). In merito a questo hanno proposto anche una classificazione dei gradi di equivalenza distinguendo tra:

a. equivalenza totale quando i due concetti sono identici;

b. equivalenza parziale quando i due concetti pur avendo molte caratteristiche in comune, ne presentano di ulteriori e diverse;

c. non equivalenza quando i concetti sono totalmente diversi.

I casi di assoluta identità concettuale corrispondenti al punto a) sono rarissimi in ambito giuridico, di conseguenza il massimo grado di equivalenza al quale può aspirare la traduzione è considerato quello di near equivalence (Sarčević, 1997).

Oggigiorno l'approccio generalmente adottato nella ricerca dell'equivalente appropriato è quello di individuare il concetto 0 l'istituto che nell' ordinamento d'arrivo riveste la medesima funzione del termine nella lingua di partenza. Questo modo di procedere consente al traduttore di trovare il significato di un determinato termine dell'ordinamento giuridico nella lingua di partenza (LdP) e renderlo accessibile nell' ordinamento giuridico della lingua di arrivo (LdA). Detto ciò in realtà questo metodo non è di facile applicazione. In primo luogo perchè seguendo questo procedimento il termine scelto dovrà contenere sia gli elementi essenziali e indispensabili per la definizione dei singoli termini sia il loro ambito semantico. In secondo luogo l'informazione documentata concernente i concetti e i termini offre la possibilità di scegliere, valutando il grado di equivalenza dei concetti appartenenti a diversi ordinamenti, la denominazione adeguata al contesto comunicativo.

Dalla comparazione di terminologie giuridiche basate su sistemi giuridici diversi vengono alla luce anche le principali difficoltà del processo di traduzione relativo all' equivalenza dei concetti.

Uno dei problemi in cui ci si imbatte spesso è che numerosi concetti e istituti tra i vari ordinamenti si differenziano fra loro. La traduzione di un termine in un altro comporta delle insidie al livello concettuale anche nei casi in apparenza semplici. Particolare attenzione richiedono i casi in cui due termini completamente identici, possono avere due significati diversi. Il termine è lo stesso, ma il concetto è diverso.

Alcuni degli esempi portati dimostrano come il termine in albanese abbia un significato diverso dal medesimo termine adoperato in un'altra lingua.

II termine decreto per esempio esiste sia nella lingua albanese sia nella lingua italiana. Nell'ordinamento italiano con questo termine vengono denominati provvedimenti di vario genere, che possono essere atti normativi, provvedimenti amministrativi o provvedimenti giurisdizionali.

Nell'ordinamento albanese il termine decreto significa un atto amministrativo a carattere normativo o nominale emanato dal Presidente della Repubblica. Da qui si può dedurre la differenza dei concetti e il fatto che il concetto in italiano include quello in albanese. II termine magistrato, in albanese, indica esclusivamente la persona investita della funzione di giudice o di pubblico ministero che ha terminato gli studi nella scuola di magistratura ${ }^{1}$. In italiano, invece, questo termine indica l'incaricato dell'amministrazione della giustizia, il giudice e allo stesso tempo designa anche alcuni uffici della pubblica amministrazione.

Un altro esempio è il termine inglese contract che concettualmente non è uguale al termine albanese kontratë. Le differenze in questo caso possono essere spiegate sulla base della contrapposizione common law/civil law. II termine albanese kontratë indica l'accordo di due o più parti a costituire, regolare 0 estinguere tra loro un rapporto giuridico patrimoniale. II termine inglese contract distingue da:

- la promise che è un elemento propedeutico alla formazione del contratto ed esprime la dichiarazione delle parti di assumersi un'obbligazione giuridica;

- l'agreement che è l'accordo tra le parti.

In questo senso contract è basato nel suo nucleo fondamentale che è il bargain: una negoziazione tra le parti in

${ }^{1}$ Nell' ordinamento giudiziario albanese la funzione di giudice e di pubblico ministero è affidato sulla base dei requisiti previsti dalla legge anche a personne che non hanno finito gli studi nella scuola di magistratura. In questo caso essi non vengono denominati magistrati. 
grado di produrre un agreement con oggetto uno scambio di promesse, suscettibile inoltre di valutazione economica.

Un caso interessante all'interno della stessa lingua è l'uso per lo stesso significato di termini diversi in base all'ordinamento giuridico di riferimento. Cosi termini completamente differenti designano un unico concetto come Prokuror i Përgjithshëm i Republikës dhe Kryeprokuror i Shtetit. Entrambi i termini indicano un magistrato della stessa qualifica che agisce nel nome dello Stato nelle cause penali. Lo stesso si può dire anche per altri termini come bashki - komunë, Këshill i Lartë i Drejtësisë dhe Këshill Gjyqësor i Kosovës, liri e organizimit dhe liri e asocimit etj. In questi casi (con riferimento alla tipologia dei casi menzionati all' interno della stessa lingua) la terminologia giuridica nelle traduzioni in lingua albanese cambia a seconda del paese di provenienza del traduttore (Albania o Kosovo).

Altri referenti (concetti e istituti) non hanno nella lingua d'arrivo i corrispettivi termini. Di conseguenza la principale difficoltà della lingua risiede nell'intraducibilità non dei termini bensì dei concetti che sono propri di alcuni sistemi giuridici e non di altri (Longinotti, 2009:256). II termine giudice viene tradotto in albanese gjykatës. Per quanto riguarda le diverse tipologie dei giudici distinte nell'ordinamento italiano (giudice di pace, giudice per le indagini preliminari, giudice dell' udienza preliminare ecc.) non vi è un corrispondente concetto nell'ordinamento albanese nonostante ciò occorre però trovare una forma linguistica per ciascuno di questi concetti dell'ordinamento italiano.

In casi come questi il traduttore giuridico deve optare per una delle seguenti scelte, vale a dire: creare nella propria lingua un nuovo termine, adottare il termine della lingua di partenza oppure descriverlo nella lingua d'arrivo².

Una particolare attenzione richiede la formazione di neologismi che hanno dato luogo a non pochi errori di traduzione presenti nei vari testi giuridici ${ }^{3}$. II termine qiradhënës anijeje in albanese viene usato come equivalente del termine italiano armatore. In realtà i due termini hanno significati diversi fra loro. Con riferimento al termine italiano questo indica chi fa navigare una nave mercantile (proprietario o noleggiatore) assumendosi l'organizzazione di persone o cose necessarie per I' utilizzazione della nave secondo la sua tipica destinazione e il rischio dell'esito negativo della navigazione. II termine albanese indica chi dà a noleggio imbarcazioni. Un altro termine, testamento olografo indica un tipo di testamento che deve essere scritto per intero, datato e sottoscritto di mano del testatore. In albanese il termine usato per la denominazione dello stesso concetto è testament me shkrim che significa testamento scritto, quindi il suo significato è più ampio.

In entrambi i casi i neologismi creati con l'intento di evitare l'uso dei prestiti attribuiscono un significato più ristretto o più ampio al termine della lingua di partenza nella lingua d'arrivo. Per questo motivo nei testi giuridici oggi si nota il ritorno all'uso dei termini armator e testament ollograf.

Per tradurre il termine giudice naturale viene usato in modo inappropriato gjykatës natyral/o i natyrshëm quando esiste già la forma linguistica - gjykatë kompetente e përcaktuar me ligj - che esprime chiaramente il concetto.

A volte il traduttore o l'interprete si limita al semplice utilizzo dei termini di lingua di partenza che se per certi casi è una soluzione inevitabile in altri è indicatore di pigrizia mentale 0 addirittura di scarsa conoscenza della terminologia giuridica. In quest'ultimo caso sono parecchi i termini che non vengono tradotti nonostante esista nella lingua d'arrivo per ciascuno di essi un termine equivalente. Abrogim al posto di shfuqizim (abrogazione), seancë "hearing" al posto di seancë dëgjimore (seduta di ascolto), draft i kushtetutës al posto di projekt i kushtetutës (progetto della costituzione), intellectual property al posto di pronësi intelektuale (proprietà intellettuale), parlament al posto di kuvend (parlamento), sono soltanto alcuni degli esempi che si incontrano spesso sia nei testi tradotti sia nella traduzione orale. Nel caso in cui i termini della lingua di partenza vengono utilizzati perché non possono essere tradotti nella lingua d' arrivo essi mantengono la grafia e il più possibile la pronuncia straniera. Dal punto di vista fonologico si osserva però che non c'è uniformità nella pronuncia di questi termini all'interno della lingua d'arrivo generando talvolta degli equivoci. In albanese si produce ad es., /fektëring/ o /faktoring/ invece di /fæktərin/ per l'inglese faktoring; /lou/ invece di /lo:/ per l'inglese law; /uelfer/ invece di / welfєə>r/ per l'inglese welfare.

Sempre per motivi di pigrizia mentale o per scarsa conoscenza della terminologia giuridica nel processo di traduzione nella lingua albanese si ricorre spesso alla traduzione letteraria dei termini generando equivoci 0

\footnotetext{
2 In pratica l'utilizzo del termine della lingua di partenza sembra essere, soprattutto nel caso di traduzione orale, la scelta abitualmente adoperata in quanto il fattore tempo rende difficile la creazione tempestiva di un neologismo e poco adatto l'impiego di perifrasi più o meno lunghe.

${ }^{3}$ Gli esempi riportati sono tratti da: M. Semini, "E drejta e detyrimeve dhe e kontratave", 1998, I. Elezi, S. Kacupi, M.Haxhia, "Komentar i Kodit Penal të Republikës së Shqipërisë", 1997; Revista "Drejtësia", 1991-1999; "Fjalor terminologjik i së drejtës", 1986, Costituzione italiana tradotta in albanese: www.immigrazioneoggi.it; www.formazioneimmigrazione.anci.it; L. Droboniku, "L'ordinamento giudiziario albanese"; C. Carazzone, "Rafforzamento delle politiche e delle azioni di lotta all'esclusione sociale minorile in Albania", www.volint.it; E. Bozheku, "Alcune riflessioni sul codice penale albanese", www.diritto.it.
} 
incompressione come nel caso quando non viene individuato il simile concetto nella lingua d'arrivo (il termine Corte d' Assise viene tradotto Gjykatë e Asizit invece di Gjykatë e Krimeve të Rënda che indica lo stesso organo giurisdizionale nell'ordinamento albanese).

Non poche difficoltà e errori di traduzione si possono rivelare anche nel caso di trasposizione dei termini dall'albanese verso un'altra lingua. Nel caso di testi tradotti in italiano il termine albanese Avokat i popullit viene tradotto con la forma letterale Avvocato del popolo o con il termine totalmente diverso giudice di pace anche se, tenendo conto della "fattispecie giuridica" indicato dal termine, il cd closest natural equivalent (Nida e Taber 1974:13) sarebbe difensore civico. Un altro termine Këshill i Lartë i Drejtësisë viene tradotto letteralmente Alto Consiglio di Giustizia nonostante il suo equivalente in italiano sia il Consiglio Superiore di Magistratura.

II termine albanese zyrë e gjendjes civile in italiano corrisponde a due termini ufficio di stato civile e ufficio di anagrafe. Quest' ultimo è il termine che viene usato nei documenti amministrativi o atti notarili tradotti in italiano tralasciando la differenza di significato fra i due termini. Anche il termine gjobë corrisponde a due diversi termini in italiano: la multa (la pena pecuniaria per i delitti) e l'ammenda (la pena pecuniaria per le contravvenzioni). In italiano spesso il termine gjobë viene tradotto multa anche quando si tratta della pena pecuniaria per le contravvenzioni.

\section{Conclusioni}

Qui sono stati messi in rilievo le difficoltà di traduzione giuridica da e per la lingua albanese (specialmente in relazione all'italiano) accennando ai problemi che nascono dal diritto e dalla lingua ma soprattutto dimostrando attraverso gli esempi portati che gli errori riscontrati derivano anche dalla mancanza in questo ambito della figura del giurista-linguista, cioè di un traduttore/ interprete che abbia buone conoscenze sia linguistiche che giuridiche. Un altro elemento che incide in maniera importante nel processo di traduzione è la mancanza degli strumenti necessari a disposizione. Si percepisce in questa situazione la necessità di effettuare studi e di pubblicazioni specializzati su questo particolare tipo di traduzione tecnica. I cambiamenti del settore in questi ultimi anni impongono con immediatezza la pubblicazione di un nuovo dizionario giuridico che tenga conto delle indicazioni degli specialisti delle singole branche del diritto e delle recentissime riflessioni degli specialisti di linguistica.

\section{References}

Arntz, R., Picht H., Mayer, F. (2004) Einführung in die Terminologiearbeit.

Cavagnoli, S., Ferrari I.E.(2009),Tradurre il diritto(Nozioni di diritto e di linguistica giuridica), CEDAM.

Cortelazzo, M.(1997), Lingua e diritto in Italia. II punto di vista dei linguisti, in La lingua del diritto. Difficoltà traduttive. Applicazioni didattiche, Atti del primo Convegno Internazionale. Milano, 5-6 ottobre 1995, Centro Linguistico dell'Università Bocconi, a cura di Leo Schena, Roma, CISU, 35-50.

Gotti, M. (1991), I linguaggi specialistici, Firenze, La Nuova Italia.

Longinotti, D.(2009), Problemi specifici della traduzione giuridica:traduzione di sentenzedal tedesco e dall'inglese, QUADERNI DI PALAZZO SERRA 17, 2009 Genova, 256-300.

Nida, E., Taber, C. R. (1974) The Theory and Practice of Translation, Lieden, Brill, 13.

Rega, L. (2000), "Aspetti e problemi della traduzione delle formule di rito nell'ambito giuridico italo-tedesco", in Daniela Veronesi (a cura di), Linguistica giuridica italiana e tedesca: obiettivi, approcci, risultati. Rechtslinguistik des Deutschen und Italienischen: Ziele, ethoden, Ergebnisse, Atti del Convegno di Studi (Bolzano, 1-3 ottobre 1998), Padova, Unipress, 449-457.

Sacco, R. (1992), Trattato di diritto comparato. Introduzione al diritto comparato,Torino, UTET.

Sarčević, S. (1997), New Approach to Legal Translation. The Hague/London/Boston: Kluwer Law International.

Tomasi, S. (2009), Il pluralismo linguistico tra identità e differenza, Rivista di Scienze della Comunicazione, 2, 81-93. 
